\title{
Using Big Data on the Benchmark Institution of Discretion in Administrative Penalty of Drug industry
}

\author{
Ning $\mathrm{Ma}^{1, *} \mathrm{Xu}$ Mengna ${ }^{2}$ \\ ${ }^{1}$ School of Law, Southeast University, Nanjing 211189,China \\ ${ }^{2}$ Municipal Hospital Affiliated of Taizhou University, Taizhou, Zhejiang Province 318099, China \\ *Corresponding author.Email: seu_law_mn@163.com
}

\begin{abstract}
In this paper, natural semantic recognition technology is used to deeply mine the data, meanwhile Regular Expression is also used to achieve text segmentation, text transformation, word segmentation, part-of-speech tagging, and delete stop words, so as to improve the accuracy of data. From the administrative penalty decision perspective, two obvious legal problems in applying the discretionary benchmark of administrative penalty cases in the drug industry were found, namely, the compliance problem and the exemption problem. On the one hand, different applicable scales and low explanatory rates cause discretionary benchmarks compliance issues; on the other hand, under the background of the replacement of old and new laws, due to the lack of normative exemption clause, some law enforcement officials will choose to directly escape the discretionary benchmark and impose a mitigated punishment below the statutory penalty based on the consideration of the cheapness of law enforcement.
\end{abstract}

Keywords: drug industry, administrative penalty, discretion benchmark, exemption clause.

\section{INTRODUCTION}

Academic circles have conducted categorization research on administrative penalty discretionary benchmarks in different fields. When setting the discretionary benchmark, two extreme cases should be prevented, oversetting and undersetting, meanwhile the appropriateness of setting the discretionary benchmark should be studied in detail[1].

Through text analysis, Hongwei Shi found that the discretionary benchmark of public security management penalty is more similar to that in criminal law[2]. Anqi Wang put forward in the field of comprehensive law enforcement of urban management that the benchmark system of administrative penalty discretion in the field of urban management as well as the crossover of responsibilities in the process of law enforcement should be improved[3]. However, there are few studies on the administrative penalty benchmark of the pharmaceutical industry.

By March 1, 2021, a total of 23,615 copies of administrative penalty decisions of the drug industry released by various provinces in China from 2010 to 2020 were collected from the Database of Wolters Kluwer. The data were cleaned and 20,517 valid samples were obtained. Then, Regular Expression is used to extract the information conforming to certain Regular Rules. Through a large number of decisions, we can see that although the specific content of each document is different, the general framework is basically the same. A general decision consists of eight parts: case number, basis of violation, basis of punishment, the content of punishment, illegal act, discretionary factor, punishment time, and punishment authority. When distinguishing these eight parts, keywords are often taken as the segmentation point. Therefore, we can extract these large parts with Regular Expression, and then carry out more detailed information extraction for each part. In addition, according to the characteristics of legal language, the lexicon of special legal terms is established, and the special legal terms in legal documents are divided into words in advance to ensure that these special terms will not be mistaken by the general word segmentation engine, to improve the accuracy of the keyword extraction system.

\section{THE OVERALL SITUATION OF THE SURVEY STATISTICS}

Among the 20,517 sample documents, there were 1,530 administrative penalty decision letters applicable to 
the discretionary benchmark, accounting for $7.46 \%$ of the total number of written decisions of administrative penalty in the pharmaceutical industry. In general, the application rate of administrative penalty discretionary provisions increased year by year, from $3.23 \%$ to $11.75 \%$. Although the applicable rate, in general, is on the rise, generally recognized by the academic circles theory that "in the process of administrative penalty shall have the priority to apply discretion benchmark instead of legal norms" is not used widely in practice. The number of cases in which executive branches apply a discretionary benchmark to measure involved circumstances and interests is small, causing obvious omission problem of discretionary datum.

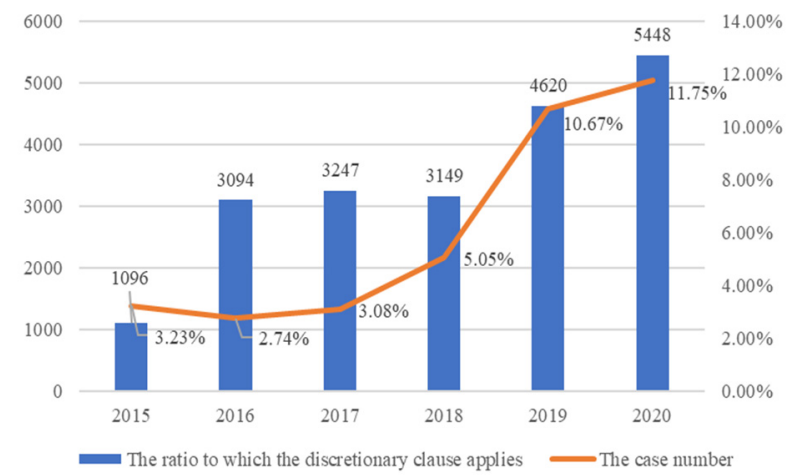

Figure 1 2015-2020 national public releasing drug class of administrative punishment cases number and the applying rate of drug administrative penalty discretion benchmark

In terms of geographical distribution, according to the geographical division of the administrative counterpart, the number of administrative penalty documents of the drug industry in Guangdong Province was 4,088, accounting for $19.9 \%$ of the total. Zhejiang province came in second with 3,734 cases, accounting for 18.2 percent of the total. In third place, Hebei Province recorded 2,909 cases of punishment documents, accounting for $9.8 \%$ of the total. For the fourth, fifth and sixth, there were 1566 cases in Shandong Province, 1,432 cases in Shanghai, and 1,302 cases in Beijing. The top 6 documents account for $68.9 \%$ of the total number of administrative punishment publicized documents. In the remaining 25 provinces, autonomous regions, and municipalities, only $31.1 \%$ of the punishment documents were published.

In the applicable administrative penalty discretion benchmark of normative documents, according to the original set by the food and drug administration in the national scope of the discretion benchmark the pharmaceutical and medical equipment administrative penalty discretion applies rules and the pharmaceutical administration law of the People's Republic of China ruled discretion standard "191 pieces of administrative punishment, accounted for $12.48 \%$; main basis for the people's government in the provincial level for drug supervision and administration department under the State Council, the provincial administrative areas in the system implementation of discretion benchmark, a total of 547 , accounted for $35.75 \%$; there are a total of 792 administrative punishment documents based on the documents independently formulated by municipal and county drug regulatory departments with districts, accounting for $51.76 \%$ of the total number of administrative penalty decisions applicable to the discretionary benchmark. In terms of the level of application of normative documents, it basically agrees with the widely recognized theory in the academic world, that is, when the administrative law enforcement agencies are faced with a complex system of discretionary benchmarks, they should give priority to the discretionary benchmarks made by the lower administrative agencies[4].

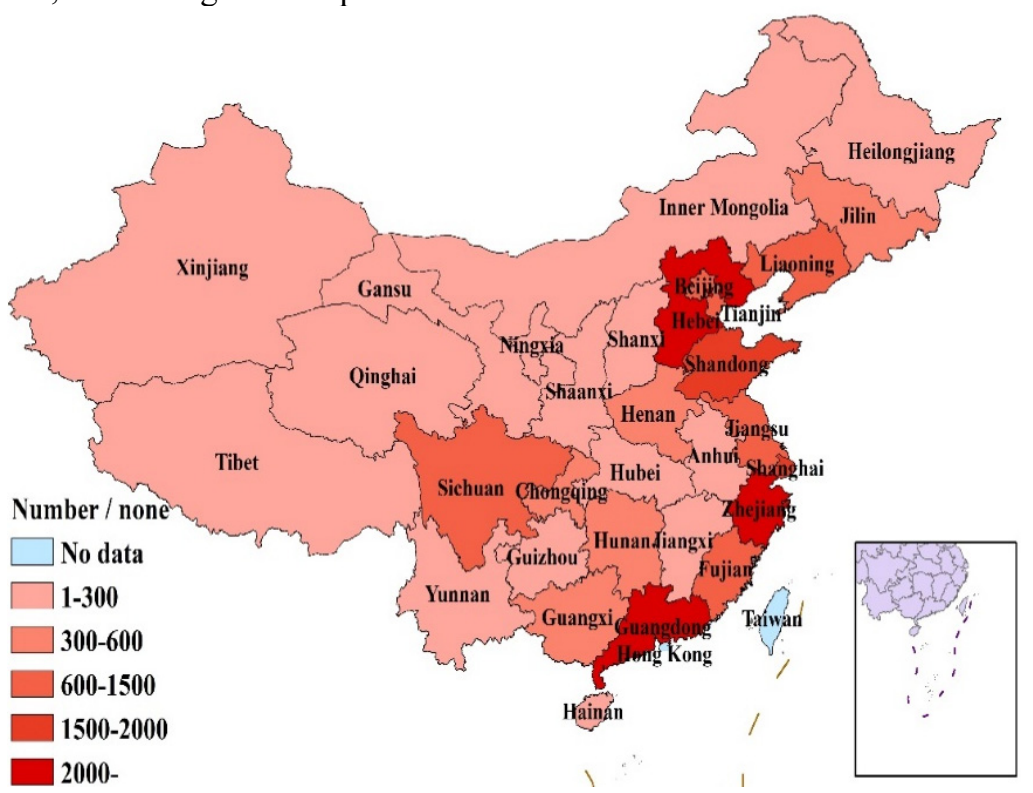

Figure 2 Total number of documents of administrative punishment for drugs published nationwide 


\section{THE COMPLIANCE PROBLEM AND THE EXEMPTION PROBLEM}

\subsection{DISCRETIONARY BENCHMARKS APPLY TO DIFFERENT SCALES}

In the application of the discretionary benchmark of administrative penalty in the drug industry, the law enforcement agencies have different scales to apply the discretionary benchmark to different administrative counterparts. Among the cases in which the penalized party was a legal entity, the discretionary standard was applied in 1,261 cases, accounting for 7 percent of the total applicable cases. In 269 cases where the penalized party was a natural person or a self-employed person, the application rate was 10.7 percent. When the penalized party is a natural person or a self-employed person, the application rate of the discretionary benchmark is nearly four percentage points higher. The differences of the scale of the discretion benchmark used may be due to law enforcers preconceived subjective impression, even in both in drug administrative penalty illegal plot and the amounts of similar cases, when the illegal behavior for a natural person or SoHo, law enforcement personnel usually preconceived think their individual behavior will not cause much influence to society, At the same time, because their weak performance ability, they should consider applying the discretionary benchmark to take a lighter or even mitigative punishment.

Different law enforcement agencies will also lead to different rates of application of discretionary benchmarks. After the institutional reform of The State Council in 2018, the primary market Supervision and Administration bureau was the main organ of administrative punishment in the drug industry, which issued 11,799 documents of administrative punishment for drugs, accounting for $57.5 \%$ of the total number of administrative punishment cases involving drugs. The number of cases applicable to the discretionary benchmark was 1256 , and the application rate of the discretionary benchmark was $10.6 \%$. The drug administrative penalties imposed by the original Food and Drug Administration were concentrated before 2018, totaling 2,353 cases. The number of cases applicable to the discretionary benchmark was 202 cases, with an application rate of 8.6 percent. After the institutional reform of The State Council, the application rate of the discretionary benchmark for administrative penalties in the drug industry increased by 2 percentage points. In stark contrast, the provincial drug administration has a low rate of application. A total of 602 drug administrative punishment decisions were made by provincial drug administrations after institutional reform, including 133 in 2019 and 469 in 2020. In 24 cases, the application rate of the discretionary benchmark was $4.0 \%$, and the application rate of the provincial drug administration was less than half of the average level.
Moreover, in the written decision of administrative penalty that applies the discretionary benchmark, some documents take the normative documents of the discretionary benchmark as one of the required items of "basis of administrative penalty" or "basis of penalty" in Administrative License and Administrative Penalty Credit Information Data Collection Publicity Standard Field Detailed Description. There are few instruments in the "facts of the offense" column that further explain how the discretionary criteria should be applied in light of the situation. According to the content of the "double publicity data standard", among the 1530 administrative penalty decision letters that have been applied to the discretionary benchmark, only 756 cases, accounting for $49.4 \%$, explained the reasons for the application of the discretionary benchmark. The remaining documents do not explain in detail whether applied discretionary benchmark or not. However, the more important the rights and interests of the relative party involved in the discretionary act are, the wider the scope of application is, and the more detailed the reasons should be explained by the law enforcer so that the administrative relative party can know the basis and reasons of the discretion from the record documents[5].

\subsection{THE ALTERNATION OF NEW AND OLD LAWS HAS LED TO AN EXEMPTION OF LIGHT DISCRETION}

The fundamental value conflict behind the dispute of discretionary benchmark exemption is the conflict between the rule and the special case. The Drug Administration Law of the People's Republic of China, newly revised in 2019, imposes stricter penalties on offenders in terms of penalty provisions for substandard drugs. In terms of fines, article 74 of the old "pharmaceutical administration law" regulate the behavior of the production, sale, and use of medicines of inferior quality, and impose a fine no less than one time and no more than three times the value of the drugs illegally produced. However, the same illegal act according to article 117 of the new "drug administration law", impose a fine of no less than 10 times and no more than 20 times the value of the drugs illegally produced or sold. If the value of drugs illegally produced or sold is less than 100,000 yuan, the value shall be calculated as 100,000 yuan; if the value of drugs illegally retailed is less than 10,000 yuan, the value shall be at least calculated as 10,000 yuan. The alternating period of the two laws has posed considerable challenges to the application of discretionary benchmarks.

In this paper, the administrative punishment case of the legal person organization in violation of the relevant regulations on drug management, the production, marketing, and sales of inferior drugs is taken as an example, and the phenomenon of the exemption of discretionary standards in this field is briefly described. 
The number of cases of substandard drugs applicable to the discretionary benchmark was 77 in 2019 and 109 in 2020 , accounting for $16.2 \%$ and $30 \%$ of the punishment cases in the corresponding years respectively. Although the application of the discretion benchmark rate increased nearly doubled, it's important to note that in the 2020 annual 376 administrative authorities substandard drugs punishment cases, 282 cases refer to the article 74 of " old pharmaceutical administration law" as legal basis. In the same time, 325 cases refer to the article 117 of "new pharmaceutical administration law" as legal basis. In other words, the number of instruments that quoted both the old and the new articles was 282 , accounting for $75 \%$. In this circumstance, the increase in the application rate of the discretionary benchmark was mainly due to the supplementary interpretation of the penalty rules that quoted the two articles at the same time. Among the remaining $70 \%$ of cases without explicit application of the normative documents of the benchmark of discretion, $31.2 \%$ of the cases exempt the benchmark of discretion, and $12.5 \%$ of the cases were given a lighter punishment with the minimum statutory penalty amount without any explanation, and $18.7 \%$ of the cases were given a lighter punishment directly below the statutory penalty amount, with no heavier punishment or maximum penalty.

In addition, due to changes in laws, local discretionary benchmarks have not yet been updated. As for the requirement of "minor violation" in the discretionary benchmark formulated by lower-level authorities, the specific effect is generally "lighter or mitigated punishment" under the statutory circumstances. However, according to the new law, when the value of goods or illegal income is less, there is a huge difference in the penalty results between lighter punishment and mitigated punishment.

To guarantee the equal and fair exercise of discretion and trust protection, the administrative organ must have reasonable reasons to make a judgment different from the discretionary benchmark[6]. In the case of the huge difference in the amount of punishment imposed by the laws before and after the revision, when the administrative law enforcement personnel refer to the applicable discretionary benchmark made following the old law, the penalty base set is too high, which may lead to the situation that the punishment decision is easy but the enforcement is difficult. In this case, local drug regulatory departments failed to construct reasonable exemption clauses in the discretionary benchmark text, which led to law enforcement personnel failing to perform the corresponding procedures in the face of individual special circumstances. Based on the consideration of the cheap of law enforcement, they usually directly escaped the discretionary benchmark and imposed a mitigated punishment below the statutory penalty.

\section{CONCLUSION}

In the application of administrative penalty discretion benchmark in the drug industry, on the one hand, the law enforcement organs apply discretion benchmark to different administrative counterparts on different scales. On the other hand, different law enforcement agencies will also cause a difference in the application rate of the discretionary benchmark. However, even in the case of applying discretionary benchmark, its explanatory rate is still low. To solve this problem, the benchmark of the discretion of drug administrative punishment should be included in the credit information data collection of administrative punishment as the "punishment basis", and as the obligation of administrative organs to exercise discretion.

In the case of a huge difference in the amount of punishment between the old and new laws, administrative law enforcement personnel refer to the application of the discretion benchmark formulated according to the old law, based on the consideration of the cheapness of law enforcement. and imposed a lighter punishment below the statutory penalty amount which directly exempt the discretion benchmark. Therefore, restrictive exemption clauses should be constructed to maintain the fairness of the case and prevent the abuse of exemption clauses from causing the omission of the discretionary benchmark.

To summarize, this paper verifies the applicability of the discretion benchmark, which has important practical significance for the administrative penalty of the drug industry. Due to the insufficient information available for the content of most cases, this paper mainly analyze two dominant datasets of legal basis and discretionary factors. Future research can consider adopting diversified research methods, such as the fusion of big data and small data research methods, to fill the gap between document data and law enforcement practice. Try to verify and correct the information behind big data by collecting small data and conducting relevant interviews.

\section{ACKNOWLEDGMENTS}

This work was funded by Soft Science Research Program of Zhejiang Province (no. 2020C35SA801366).

\section{REFERENCES}

[1] Y. Chen, "The appropriateness of setting the discretionary benchmark of Administrative Penalty -- Take Zhejiang province as the observation point in recent years," Administrative Tribune, vol.3,p.65,2010.

[2] H.Shi.(2018) Research on discretionary Benchmark of public security Administration Punishment. Xiangtan University Publishing, Xiangtan.pp.43. 
[3] A.Wang.(2018) The benchmark system of administrative penalty discretion in the field of urban management. Yangzhou University Publishing, Yangzhou.pp.17.

[4] Y.Zhou, L.Zhou, "On the relativity of the effect of discretionary Benchmark and its selecting and applying," Administrative Law Review, vol. 2, pp. 3-13,2018.

[5] G.Wang. (2016) Structure and Review of Administrative Discretion. China People's University Press Publishing, Beijing.pp.86.

[6] Y.Zhou. (2015) Study on the benchmark of administrative discretion. China People's University Press Publishing, Beijing.pp.76. 